\title{
Imaging of Weak-Source Distributions in LSO-Based Small-Animal PET Scanners
}

\author{
Andrew L. Goertzen ${ }^{1,2}$, Joon Young Suk ${ }^{2}$, and Christopher J. Thompson ${ }^{2}$ \\ ${ }^{1}$ Department of Radiology, University of Manitoba, Winnipeg, Manitoba, Canada; and ${ }^{2}$ McConnell Brain Imaging Center, \\ Montreal Neurological Institute, Montreal, Quebec, Canada
}

\begin{abstract}
Lutetium oxyorthosilicate (LSO)- or lutetium-yttrium oxyorthosilicate (LYSO)-based PET scanners have intrinsic radioactivity in the scintillator crystals due to the presence of ${ }^{176} \mathrm{Lu}$, which decays by $\beta$-emission followed by one or more prompt $\gamma$-ray emissions. This leads to intrinsic true counts that can influence the image when scanning low levels of activity. An evaluation of the effects of this intrinsic activity for low levels of activity and different energy windows is performed on an LSO-based small-animal PET scanner. Methods: Intrinsic count rate and sensitivity were measured for a range of lower-level discriminators (LLDs) ranging from 100 to $750 \mathrm{keV}$. The noise equivalent count rate (NECR) as a function of LLD for activity levels from $100 \mathrm{~Bq}$ to $100 \mathrm{kBq}$ was estimated using a combination of measurement and previously published data for this scanner. Phantom imaging was performed using three ${ }^{68} \mathrm{Ge}$ sources of strength 55 , 220 , and $940 \mathrm{~Bq}$ and LLD levels of 250,350 , and $400 \mathrm{keV}$. The images were assessed using a contrast-to-noise ratio (CNR) analysis and by comparing the observed ratio of source activities to the true ratio value. Results: The intrinsic true count rate is reduced from 940 counts per second (cps) for a 250- to 750-keV energy window to $<2 \mathrm{cps}$ for a 400 - to $750-\mathrm{keV}$ window. There is a corresponding 2-fold drop in sensitivity for detected true events for external positron sources for these 2 energy windows. The NECR versus LLD curves showed a highly peaked shape, with the optimum LLD being approximately $425 \mathrm{keV}$. The phantom image results were dominated by the intrinsic true counts when an energy window of $250-750 \mathrm{keV}$ was used. The intrinsic true counts were almost completely removed by raising the LLD to $400 \mathrm{keV}$. The CNR for each of the sources was higher for the narrow energy window and the $55 \mathrm{~Bq}$ could be easily visualized in images acquired with LLD levels of 350 and $400 \mathrm{keV}$ but not when the $250-\mathrm{keV}$ LLD was used. Images acquired with an LLD of $400 \mathrm{keV}$ and reconstructed with 2-dimensional filtered backprojection were the most quantitatively accurate. Conclusion: It is possible to visualize sources of $<1 \mathrm{kBq}$ in LSO-based animal PET systems by raising the LLD to $400 \mathrm{keV}$ to exclude the majority of the counts due to the intrinsic activity present in the LSO.
\end{abstract}

Key Words: lutetium oxyorthosilicate; intrinsic activity; PET; energy window; small-animal PET

J Nucl Med 2007; 48:1692-1698

DOI: 10.2967/jnumed.107.040584

Received Feb. 7, 2007; revision accepted Jul. 11, 2007

For correspondence or reprints contact: Andrew L. Goertzen, PhD, Department of Radiology, University of Manitoba, John Buhler Research Centre, 751-715 McDermot Ave., Winnipeg, Manitoba, R3E 3P4, Canada.

E-mail:agoertzen2@hsc.mb.ca

COPYRIGHT @ 2007 by the Society of Nuclear Medicine, Inc.
$\mathbf{P}$ ET scanner performance is usually optimized for a specific imaging task based on the results of noise equivalent count rate (NECR) measurements $(1-4)$. The usual measure reported is the peak NECR value and the activity at which the peak occurs and, in general, the energy window is chosen to maximize the value of the peak NECR. When the imaging task involves very low levels of injected activity, such as cell trafficking studies (5) or gene expression imaging (6), the scanner is operating at a count rate far less than the peak NECR value.

When low activity levels are being imaged, there is a very limited number of true counts that can be acquired so that one would consider using a wider energy window to increase the sensitivity (7). However, the choice of energy window at low activity levels is complicated for PET scanners that use lutetium-based scintillators such as lutetium oxyorthosilicate (LSO) because of the intrinsic radioactivity present in the scintillator $(8-10)$. The lutetium used in LSO contains $2.6 \%{ }^{176} \mathrm{Lu}$, which decays by $\beta^{-}$decay (mean $\beta^{-}$-energy of $420 \mathrm{keV}$ ). As shown in Figure 1, the $\beta^{-}$-decay is followed by the prompt emission of $\gamma$-rays of energy $307 \mathrm{keV}$ (94\%), $202 \mathrm{keV}(78 \%)$, and $88 \mathrm{keV}$ (15\%) $(11)$. The $\beta^{-}$-particle is detected in the crystal in which it was emitted but the $\gamma$-ray may escape and be detected in another crystal, causing a valid coincident count that lacks the collinearity possessed by the $511-\mathrm{keV}$ photons associated with positron decay. The counts originating for the decay of ${ }^{176} \mathrm{Lu}$ are uniformly spread out across the field of view (FOV) such that there is a resemblance to a dataset acquired without correction for random coincidences.

If the lower energy threshold is lowered to increase the sensitivity, there will be a large increase in the number of accepted $\gamma$-rays originating in the LSO. As pointed out by Yamamoto et al. (10), the effects of the intrinsic activity will be larger for animal systems than for clinical systems because of the lower energy thresholds used. Because the effects of the intrinsic activity are minor for large activity levels, an energy window of $250-750 \mathrm{keV}$ is commonly used for small-animal PET systems. This choice is made because the peak value of the NECR curve is higher for this energy window than for narrower energy windows (12). 


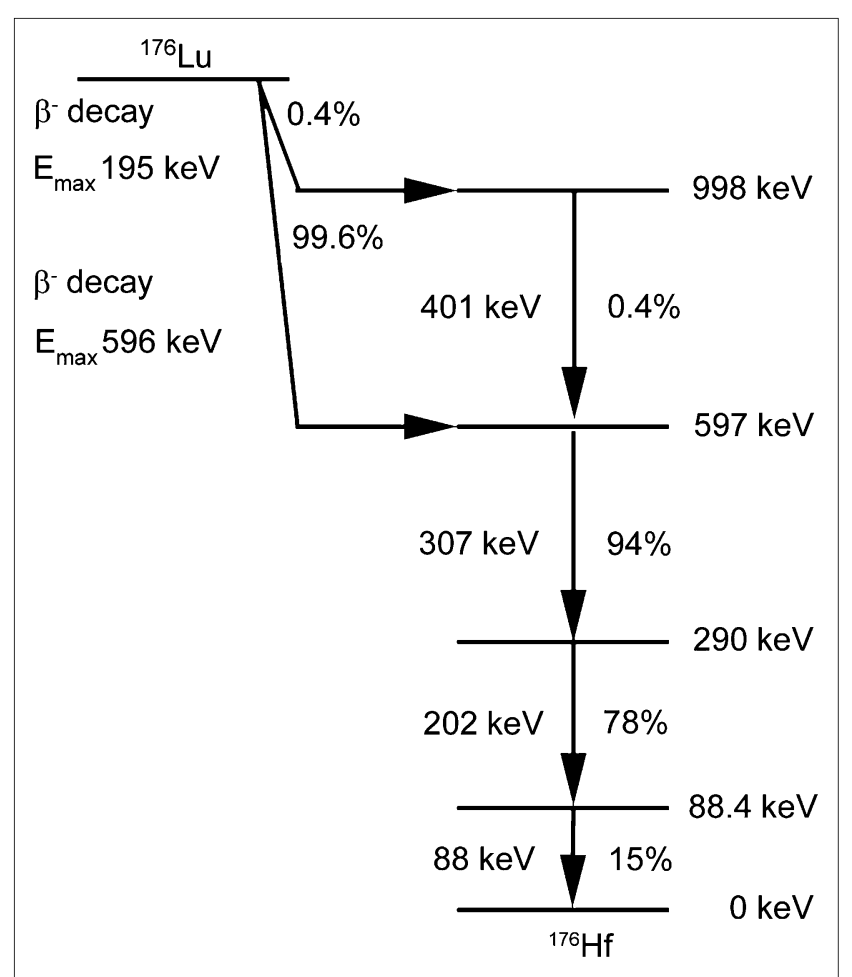

FIGURE 1. Decay scheme of ${ }^{176} \mathrm{Lu}$ showing $\beta^{-}$-emission followed by cascade of prompt $\gamma$-ray emissions. All intensities are relative to number of decays of ${ }^{176} \mathrm{Lu}$. Figure is drawn using data from (11). $E_{\max }=$ maximum energy.

However, when low amounts of radioactivity are present in the scanner FOV, the intrinsic true counts can contribute a considerable fraction of the counts being detected. For example, in the microPET R4 (Siemens Preclinical Solutions), the activity of ${ }^{176} \mathrm{Lu}$ in the $271 \mathrm{~cm}^{3}$ of LSO is approximately $75 \mathrm{kBq}$, but because of the near $100 \%$ efficiency for detecting the emitted $\beta^{-}$-particle, this creates a coincident count rate equivalent to having a positron emitter with approximately $370 \mathrm{kBq}$ in the scanner FOV when an energy window of 250-750 keV is used (13).

In this article we seek to answer the question: "Is the energy window chosen to optimize the peak NECR at high activity levels also the best choice for operation at very low count levels?" System count rates and sensitivity are measured for a range of energy windows. These measurements, in combination with previously published data for the microPET R4 scanner, allow us to estimate the NECR at low count rates as a function of energy window. A phantom with three ${ }^{68} \mathrm{Ge}$ sources, each with activity of $<1 \mathrm{kBq}$, was imaged using several energy windows, and the images were evaluated using a contrast-to-noise ratio (CNR) analysis.

\section{MATERIALS AND METHODS}

The scanner used for all measurements is the LSO-based microPET R4 located at the Montreal Neurological Institute.

\section{Intrinsic Count Rate Versus Energy Window}

The intrinsic coincident count rate due to the decay of ${ }^{176} \mathrm{Lu}$ in the LSO was measured for a range of energy windows by fixing the upper-level discriminator (ULD) at $814 \mathrm{keV}$ and varying the lower-level discriminator (LLD) in steps of approximately $6.5 \mathrm{keV}$ ranging from $100 \mathrm{keV}$ up to $750 \mathrm{keV}$. For each energy window, a 3-min scan was performed with no external activity near the scanner. The intrinsic count rate was measured by recording the number of histogrammed prompts, randoms, and true counts in the sinograms.

\section{System Sensitivity Versus Energy Window}

The relative system sensitivity was assessed for the same energy windows as were used to measure the intrinsic count rate by placing a $2.4-\mathrm{MBq}, 183$-mm-long ${ }^{68} \mathrm{Ge}$ line source in the center of the scanner and acquiring data for 3 min per energy window. The measured count rate was corrected for intrinsic activity events by subtracting the values recorded from the intrinsic count rate measurements with similar energy windows. The sensitivity for a given LLD relative to that with an LLD of $250 \mathrm{keV}, \mathrm{S}_{\text {rel }}(\mathrm{LLD})$ was calculated as:

$$
\mathrm{S}_{\mathrm{rel}}(\mathrm{LLD})=\frac{\text { Sensitivity }(\text { LLD })}{\text { Sensitivity }(\mathrm{LLD}=250 \mathrm{keV})} . \quad \text { Eq. } 1
$$

\section{Coincidence System Energy Spectra}

True coincident events detected after the decay of ${ }^{176} \mathrm{Lu}$ are due to the detection of a $\beta$-particle in the crystal where the decay happened and a $\gamma$-ray in a second crystal. If an energy spectrum is acquired in singles mode, it will be dominated by the energy spectrum of the $\beta$-particle because of the near $100 \%$ detection efficiency for this particle. To estimate the energy spectrum due to the ${ }^{176} \mathrm{Lu}$ decay as measured by the microPET R4 system under normal operating conditions the count rate of the system was measured as the energy window was varied. The ULD was fixed at $812 \mathrm{keV}$ and the LLD varied in steps of $6.5 \mathrm{keV}$ over a range of $100-750 \mathrm{keV}$. For each energy window, a 3-min acquisition was performed with no external source in the FOV and the number of true counts in the sinogram was recorded, with true counts representing the prompt counts corrected for randoms using a delayed coincidence window. The difference in true counts between 2 successive LLD settings was taken as the counts in the energy bin corresponding to the given LLD level. This spectrum measurement process was repeated with the $2.4-\mathrm{MBq}{ }^{68} \mathrm{Ge}$ line source centered in the FOV to provide a comparison of the intrinsic energy spectrum with that due to a positron-emitting source.

\section{Estimate of NECR Versus LLD}

The NECR versus LLD was estimated for low activity levels for mouse- and rat-sized phantoms according to the usual formulation for NECR (14):

$$
\mathrm{R}_{\mathrm{NEC}}(\mathrm{LLD})=\frac{\mathrm{R}_{\mathrm{t}}^{2}(\mathrm{LLD})}{\mathrm{R}_{\mathrm{TOT}}(\mathrm{LLD})}
$$

where $R_{t}(L L D)$ is the true count rate, $R_{T O T}(L L D)$ is the total prompt count rate, and $\mathrm{R}_{\mathrm{NEC}}(\mathrm{LLD})$ is the NECR, with all parameters 
being functions of the LLD. Because of the poor statistical quality of any measurement made at very low activity levels, the NECR estimate was based on estimating both $\mathrm{R}_{\mathrm{t}}(\mathrm{LLD})$ and $\mathrm{R}_{\mathrm{TOT}}$ (LLD) from a combination of measurements at higher count rates and from data published by Knoess et al. regarding the performance of the microPET R4 scanner (12).

The total prompt count rate, $\mathrm{R}_{\mathrm{TOT}}(\mathrm{LLD})$, was estimated as the total of the prompts due to $\beta^{+}$-emission events, $R_{P, \beta}(L L D)$, plus the count rate due to the intrinsic prompt count rate, $R_{P, \text { int }}(L L D)$, or:

$$
\mathrm{R}_{\mathrm{TOT}}(\mathrm{LLD})=\mathrm{R}_{\mathrm{P}, \beta}(\mathrm{LLD})+\mathrm{R}_{\mathrm{P}, \text { int }}(\mathrm{LLD}) \times \mathrm{k} \times \mathrm{f}, \quad \text { Eq. } 3
$$

where $\mathrm{k}$ represents the fraction of the FOV covered by the phantom and $\mathrm{f}$ is a factor that accounts for the fact that the intrinsic counts have a slight spatial dependence. For the $60-\mathrm{mm}-$ diameter rat-sized phantom, $\mathrm{k}$ has a value of 0.6 , whereas for the 30 -mm-diameter mouse-sized phantom $\mathrm{k}$ is 0.3 . The intrinsic true count rate was approximately $25 \%$ lower in the middle of the FOV as compared with the edge of the FOV. This spatial dependence was well modeled using a sine function. The value of $\mathrm{f}$ for the 60 -mm-diameter rat-sized phantom was 0.944 , whereas for the 30-mm-diameter mouse-sized phantom f was 0.918. Whereas $\mathrm{R}_{\mathrm{P} \text {,int }}(\mathrm{LLD})$ could be calculated from the measurements of intrinsic count rate versus energy window, $R_{P, \beta}(L L D)$ was estimated according to the formula:

$$
\mathrm{R}_{\mathrm{P}, \beta}(\mathrm{LLD})=\mathrm{A} \times \mathrm{S}_{\mathrm{rel}}(\mathrm{LLD}) \times \operatorname{Sensitivity}(\mathrm{LLD}=250 \mathrm{keV}),
$$

with $A$ being the activity in the scanner FOV, $S_{\text {rel }}$ (LLD) given by Equation 1, and sensitivity at an LLD of $250 \mathrm{keV}$ being that reported by Knoess et al., $2.18 \%$. The true count rate was then estimated as:

$$
\mathrm{R}_{\mathrm{t}}(\mathrm{LLD})=\mathrm{R}_{\mathrm{P}, \beta}(\mathrm{LLD}) \times\{1-\mathrm{SF}(\mathrm{LLD})\}, \quad \text { Eq. } 5
$$

where $\mathrm{SF}(\mathrm{LLD})$ is the scatter fraction as a function of LLD level and is estimated by a linear fit to the scatter fraction data of Knoess et al. for their rat- and mouse-sized phantoms. The scatter fraction versus LLD value exhibited a very linear behavior over the LLD range of $250-450 \mathrm{keV}$, with an $R^{2}$ value of 0.9971 for the rat phantom and 0.9979 for the mouse phantom.

The contribution of randoms to $\mathrm{R}_{\mathrm{TOT}}$ is largely ignored in this NECR calculation; however, this is a reasonable assumption because the randoms due to the intrinsic activity are accounted for in $\mathrm{R}_{\mathrm{P} \text {,int }}$, and a very small amount of activity placed in the FOV will only negligibly increase the singles rate and, hence, negligibly increase the randoms rate. NECR estimates were made for activity levels of $100 \mathrm{~Bq}, 1 \mathrm{kBq}, 10 \mathrm{kBq}$, and $100 \mathrm{kBq}$.

\section{Phantom Imaging}

A weak-source phantom was constructed using three $1-\mathrm{cm}^{3}$ ${ }^{68} \mathrm{Ge}$ aliquots (supplied with past ${ }^{68} \mathrm{Ge}$ cylinder orders) secured to the outside of a paper roll. The source strengths were 55, 220, and $940 \mathrm{~Bq}$. The phantom was scanned for $1 \mathrm{~h}$ each using LLD settings of 250,350, and $400 \mathrm{keV}$. The ULD was held constant at $750 \mathrm{keV}$. A transmission scan was performed using a ${ }^{57} \mathrm{Co}$ source and was used to verify source locations. The emission images were reconstructed with the manufacturer's software using Fourier rebinning followed by either 2-dimensional filtered backprojection
(2D-FBP) or 2-dimensional ordered-subsets expectation maximization (2D-OSEM) with a voxel size of $0.845 \times 0.845 \times 1.21 \mathrm{~mm}^{3}$. Attenuation correction was applied using the measured attenuation sinogram. Scatter correction was not applied to the data because the sinograms had too few counts for the manufacturer's scatter correction to be reliably applied. Postreconstruction gaussian smoothing of $3.38 \mathrm{~mm}$ in the transverse direction and $3.63 \mathrm{~mm}$ in the axial direction was applied. Images were examined qualitatively for the effects of the intrinsic activity as well as quantitatively using a region-of-interest (ROI) analysis. ROIs were drawn with the aid of the transmission image over 5 slices in each source region as well as in 4 background regions in each of the 5 slices. The CNR was calculated for each source and LLD setting as:

$$
\mathrm{CNR}=\frac{\left(\mathrm{S}_{\mathrm{ROI}}-\mathrm{S}_{\text {Background }}\right)}{\sqrt{\left(\sigma_{\text {ROI }}^{2}+\sigma_{\text {Background }}^{2}\right) / 2}},
$$

where $S_{\text {ROI }}$ and $\sigma_{\text {ROI }}$ are the mean signal and $S D$, respectively, in the ${ }^{68} \mathrm{Ge}$ source, and $\mathrm{S}_{\mathrm{Background}}$ and $\sigma_{\text {Background }}$ are the mean signal and SD in the background ROI region. Because the SD is different in $\mathrm{S}_{\mathrm{ROI}}$ and $\mathrm{S}_{\mathrm{Background}}$, the noise is represented as the square root of the average variance. The quantitative accuracy of each image was assessed by comparing the mean values of the ROIs drawn for the $220-\mathrm{Bq}$ and $55-\mathrm{Bq}$ sources to the mean value of the ROI drawn for the $940-\mathrm{Bq}$ source. The percentage error was calculated for the 220-Bq and 55- $\mathrm{Bq}$ sources as:

$$
\% \text { error }=100 \times\left(\frac{\frac{\mathrm{ROI}_{\mathrm{i}}}{\mathrm{ROI}_{940 \mathrm{~Bq}}}-\frac{\mathrm{A}_{\mathrm{i}}}{940 \mathrm{~Bq}}}{\frac{\mathrm{A}_{\mathrm{i}}}{940 \mathrm{~Bq}}}\right), \quad \text { Eq. } 7
$$

where $\mathrm{ROI}_{\mathrm{i}}$ is the mean value in the ROI for the source being examined, $\mathrm{ROI}_{940 \mathrm{~Bq}}$ is the mean value in the ROI for the $940-\mathrm{Bq}$ source, and $A_{i}$ is the activity in the source.

\section{RESULTS}

\section{Intrinsic Count Rate Versus Energy Window}

Figure 2 shows the intrinsic true count rate versus LLD setting. The intrinsic prompts count rate was very similar to the true count rate because even at the lowest LLD setting of $100 \mathrm{keV}$, the randoms count rate was only $11 \mathrm{cps}$ as compared with the true count rate of $2,500 \mathrm{cps}$. It can be seen in the plot that the intrinsic count rate drops rapidly as the LLD is raised to exclude the $202-\mathrm{keV}$ and $307-\mathrm{keV}$ $\gamma$-rays from ${ }^{176} \mathrm{Lu}$ but changes very little above $400 \mathrm{keV}$.

\section{System Sensitivity Versus Energy Window}

Figure 3 shows the system sensitivity versus LLD setting for the ${ }^{68} \mathrm{Ge}$ line source after correction for the intrinsic count rate. All sensitivities are relative to the value for a 250-keV LLD setting. The data show that as the LLD is raised from 250 to $400 \mathrm{keV}$, the sensitivity drops by a factor of approximately 2 .

\section{Coincidence Energy Spectra Versus Energy Window}

Figure 4 shows the coincidence energy spectra acquired for the $2.4-\mathrm{MBq}^{68} \mathrm{Ge}$ line source and for no external source 


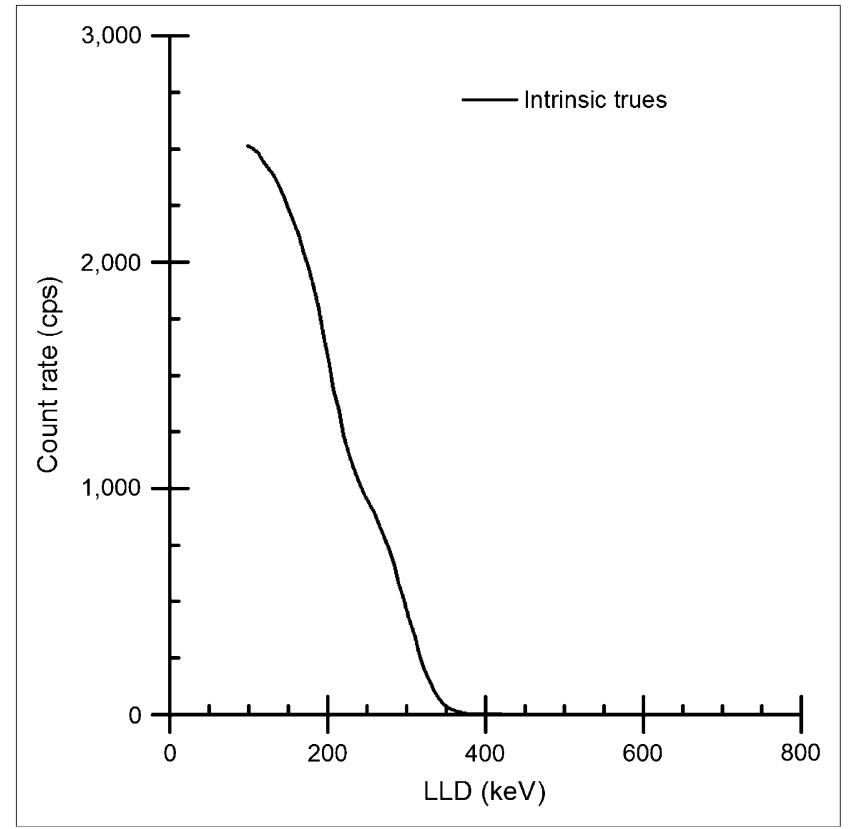

FIGURE 2. Intrinsic true count rate in the microPET R4 due to ${ }^{176} \mathrm{Lu}$ as a function of LLD. The ULD was fixed at $814 \mathrm{keV}$.

in the FOV. The spectra show that the counts due to the intrinsic ${ }^{176} \mathrm{Lu}$ activity are not negligible for low LLD settings but that the intrinsic activity contribution is minimal when an LLD of $>400 \mathrm{keV}$ is used.

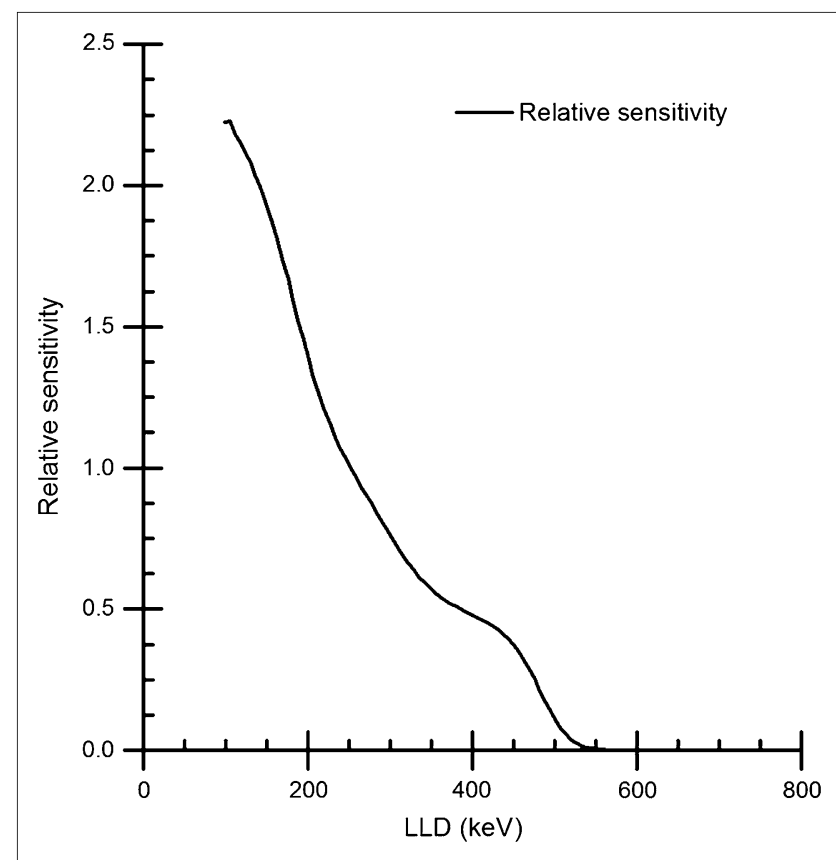

FIGURE 3. Sensitivity of microPET R4 for $2.4-\mathrm{MBq}{ }^{68} \mathrm{Ge}$ line source vs. LLD setting. All sensitivities are relative to an LLD setting of $250 \mathrm{keV}$.

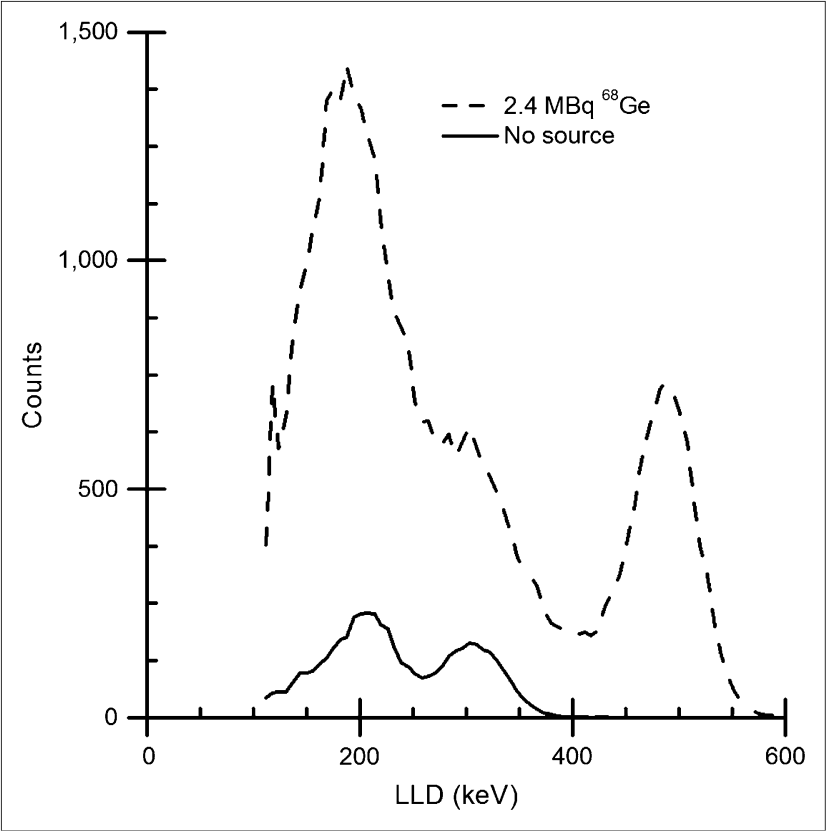

FIGURE 4. Coincidence energy spectra for microPET R4 acquired by fixing the ULD at $814 \mathrm{keV}$ and varying the LLD in steps of $6.5 \mathrm{keV}$. Values plotted represent difference in counts between 2 successive LLD settings.

\section{NECR Versus LLD}

Figure 5 shows the estimates of NECR for activity levels ranging from $100 \mathrm{kBq}$ down to $100 \mathrm{~Bq}$ for rat- and mousesized objects. The peak NECR value for the rat phantom, as a fraction of positron emission events, was $0.742 \%$ at 425 $\mathrm{keV}$ for $100 \mathrm{kBq}, 0.730 \%$ at $425 \mathrm{keV}$ for $10 \mathrm{kBq}, 0.623 \%$ at $425 \mathrm{keV}$ for $1 \mathrm{kBq}$, and $0.257 \%$ at $433 \mathrm{keV}$ for $100 \mathrm{~Bq}$. For the mouse phantom, the corresponding values were $1.266 \%$ at $144 \mathrm{keV}$ for $100 \mathrm{kBq}, 0.867 \%$ at $380 \mathrm{keV}$ for $10 \mathrm{kBq}$, $0.784 \%$ at $425 \mathrm{keV}$ for $1 \mathrm{kBq}$, and $0.444 \%$ at $425 \mathrm{keV}$ for $100 \mathrm{~Bq}$. It can be seen that the NECR versus LLD setting has a very highly peaked shape at low activity levels, indicating that minor changes to the LLD setting can exclude large amounts of the intrinsic counts and have a profound effect on the noise equivalent counts being acquired. The relative impact of the intrinsic counts for different activity levels can be seen by looking at the value of the peak NECR. As activity levels decrease so does the fraction of $\beta^{+}$-events that create a noise equivalent count, with this reduction being most noticeable as the activity level drops below $1 \mathrm{kBq}$.

\section{Phantom Imaging Results}

Figure 6 shows the emission images of the weak-source phantom. When an LLD of $250 \mathrm{keV}$ is used, the artifacts from the intrinsic activity dominate the image; however, when the LLD is raised to values of $350 \mathrm{keV}$ or higher it becomes possible to resolve all 3 sources. The counts due to intrinsic activity are distributed relatively uniformly across the FOV, creating a direct current (DC) type offset in the sinograms similar to that seen from an uncorrected 
FIGURE 5. NECR vs. LLD for activity levels of $100 \mathrm{kBq}(\mathrm{A}), 10 \mathrm{kBq}(\mathrm{B}), 1 \mathrm{kBq}$ $(\mathrm{C})$, and $100 \mathrm{~Bq}(\mathrm{D})$ in rat and mouse phantoms. At lower activity levels, intrinsic activity has an increasing effect as seen by the increasingly peaked shape of the NECR curve as well as the lower relative value of the peak NECR value.
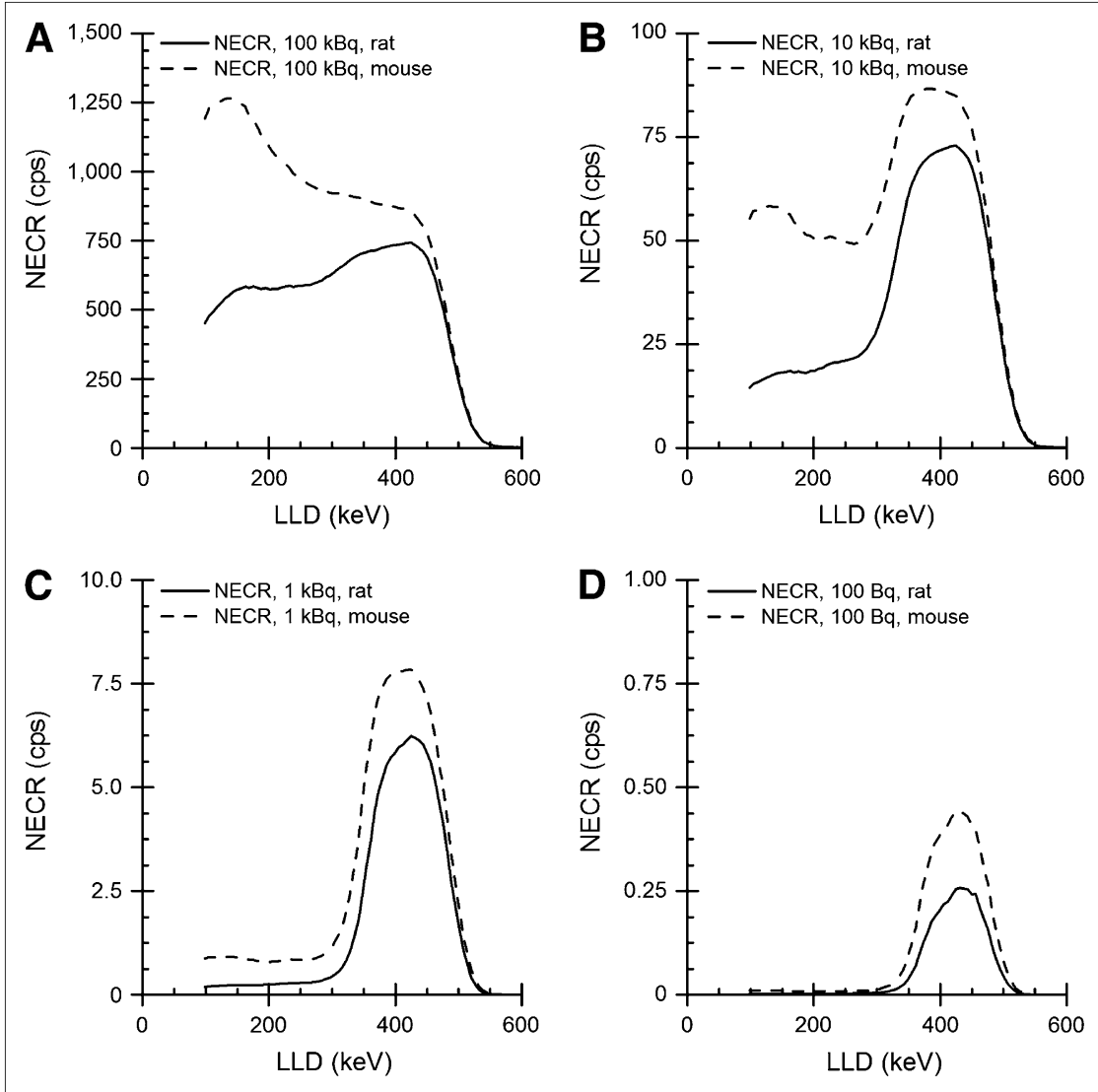

randoms background. This DC offset leads to the strong ring artifact seen in the $250-$ to $750-\mathrm{keV}$ window images. CNR values for each of the reconstructions are given in Table 1. There is an improvement in CNR for all sources when the LLD is increased to $400 \mathrm{keV}$, consistent with the results of the NECR versus LLD calculations. Table 2 lists the percentage error in the ratio of the ROI measurements for each source. The results show that the most quantitatively accurate images are obtained for 2D-FBP reconstructions of data acquired with a $400-\mathrm{keV}$ LLD value. For this case, all errors are $<5 \%$. In comparison, the smallest error observed for a $250-\mathrm{keV}$ LLD setting was $>25 \%$.

\section{DISCUSSION}

The results suggest that when very weak sources are being imaged in an LSO-based scanner the image quality degrades considerably due to the intrinsic activity when a wide energy window is used. The amount of true counts due to the decay of ${ }^{176} \mathrm{Lu}$ increases rapidly as the LLD setting is lowered below $400 \mathrm{keV}$. NECR calculations for weak activity levels show that the NECR versus LLD curve has a highly peaked shape at activity levels below $100 \mathrm{kBq}$, with an optimum setting for the LLD being approximately $425 \mathrm{keV}$. The results of the phantom imaging were consistent with the NECR calculations, with the CNR being improved for sources ranging from 55 to $940 \mathrm{~Bq}$ when an
LLD of $400 \mathrm{keV}$ was used instead of $250 \mathrm{keV}$. These results indicate that, despite the $50 \%$ reduction in sensitivity from raising the LLD from 250 to $400 \mathrm{keV}$, the image quality improves because of the reduction in the intrinsic count rate. The best quantitative accuracy observed was for images acquired with an LLD of $400 \mathrm{keV}$ and reconstructed with 2D-FBP. For this case, the ratio of measured source activities in the images was accurate to within $5 \%$ of the true ratio values.

An initial alternative approach we considered for dealing with the intrinsic count rate was to scan using a wider energy window and perform a postacquisition subtraction of the intrinsic counts from the data. The problem with this approach is that the intrinsic counts will still contribute statistical noise to the data by contributing to $\mathrm{R}_{\mathrm{TOT}}$ in Equation 2, in a similar manner as random coincidences that are subtracted yet still influence the statistical noise. Thus, performing a subtraction to correct for the intrinsic activity will not improve the noise equivalent counts that are acquired. As a result, the issue of the intrinsic activity must be addressed at the acquisition phase rather than the postprocessing phase.

Finally, the results presented here demonstrate that it is critical that the energy window used for acquiring images must be optimized to the imaging task. The wide energy windows such as $250-750 \mathrm{keV}$ used at high activity levels are not useful when one is attempting to visualize sources 


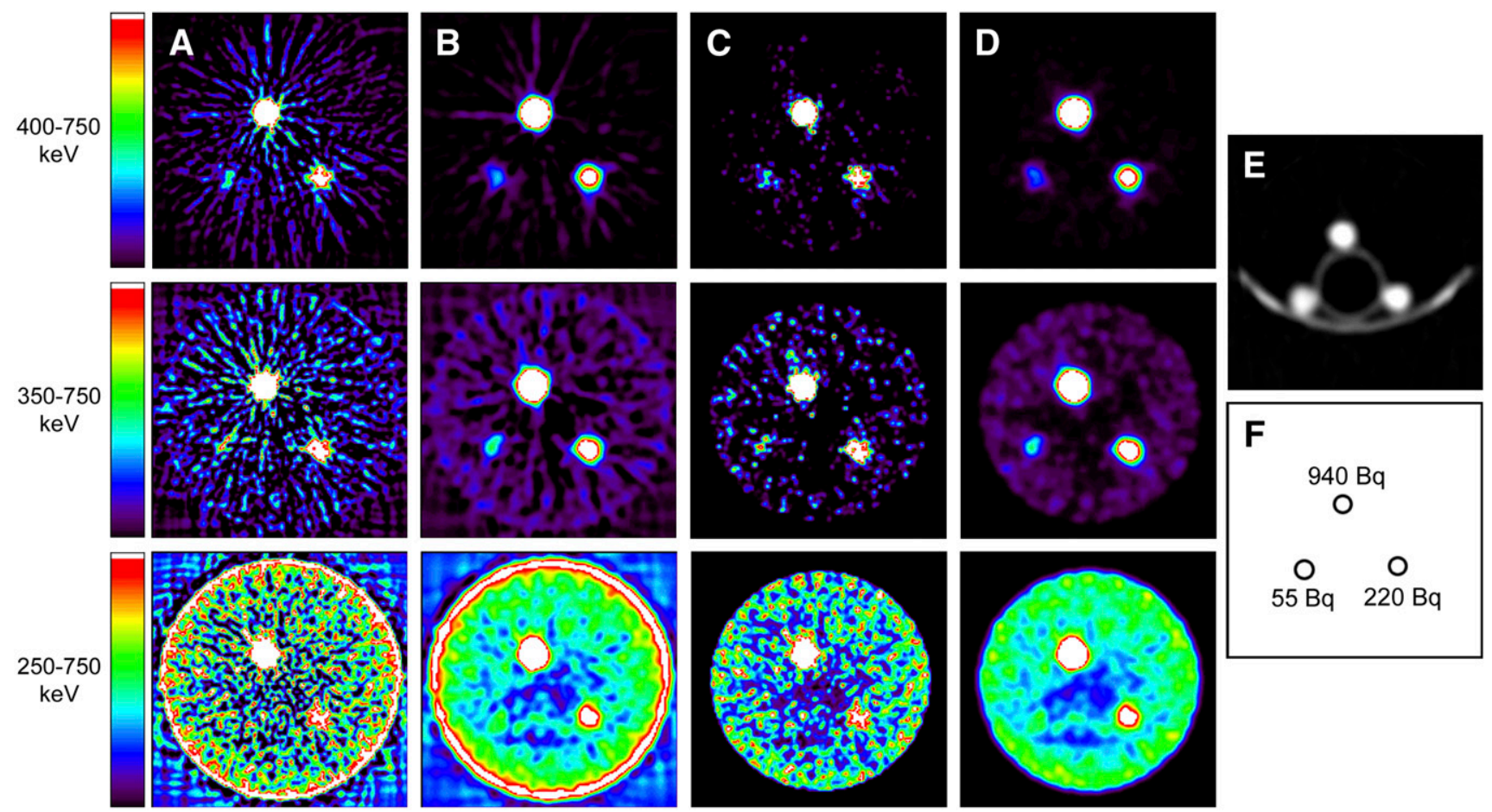

FIGURE 6. Phantom imaging results for 1-h acquisitions of 3 sources of strength: $940 \mathrm{~Bq}, 220 \mathrm{~Bq}$, and $55 \mathrm{~Bq}$ for LLD settings of 250, 350, and $400 \mathrm{keV}$. Reconstruction algorithms used were 2D-FBP (A), 2D-FBP with postreconstruction smoothing (B), 2DOSEM (C), and 2D-OSEM with postreconstruction smoothing (D). Transmission image is shown in E and a schematic of source locations is shown in F. Note reduction in background as value of the LLD is raised. All images are scaled such that maximum intensity is at mean value of the ROI drawn for the $220-\mathrm{Bq}$ source.

that are only a few tens of kilobecquerels or less in strength. Although these results were acquired using a microPET R4, they are applicable to any scanners of the microPET family. Indeed, because the R4 system has the least LSO of any of the microPET scanners, it can be assumed that the intrinsic count rate will be higher for the other scanners and, thus, the effects of the intrinsic activity even greater.

\section{CONCLUSION}

This study evaluated the performance of a microPET R4 scanner for imaging very low levels of activity. Our results indicate that when scanning at low activity levels the acquisition protocol must be tailored to match the amount of activity being used. With an appropriate choice of LLD of $400 \mathrm{keV}$, it was possible to visualize a $55-\mathrm{Bq}{ }^{68} \mathrm{Ge}$ source in the scanner with a 1-h scan. This source was not visible when an LLD setting of $250 \mathrm{keV}$ was used. At low activity levels the NECR versus LLD has a highly peaked shape, with an optimum value for the LLD being $425 \mathrm{keV}$ for activities under $100 \mathrm{kBq}$. For images acquired with an LLD of $400 \mathrm{keV}$ and reconstructed with 2D-FBP, the measured ratios of activity between sources agreed to within 5\% of their true values. A simple subtraction method will not be suitable for correcting the data because the statistical noise due to the intrinsic trues will still be present in the total count rate in a manner very analogous to how randoms contribute statistical noise even when the randoms correction is noiseless. Thus, it is critical that the energy window be set such that the maximum amount of intrinsic counts are excluded.

TABLE 1

CNRs for Weak-Source Phantom Images

\begin{tabular}{|c|c|c|c|c|c|c|c|c|c|c|}
\hline \multirow[b]{3}{*}{ Reconstruction } & \multirow[b]{3}{*}{ Smoothed } & \multicolumn{3}{|c|}{ 940-Bq source } & \multicolumn{3}{|c|}{ 220-Bq source } & \multicolumn{3}{|c|}{$55-\mathrm{Bq}$ source } \\
\hline & & \multicolumn{3}{|c|}{ LLD (keV) } & \multicolumn{3}{|c|}{ LLD (keV) } & \multicolumn{3}{|c|}{ LLD (keV) } \\
\hline & & 250 & 350 & 400 & 250 & 350 & 400 & 250 & 350 & 400 \\
\hline 2D-FBP & No & 3.44 & 3.10 & 5.63 & 1.29 & 1.95 & 2.99 & 0.20 & 0.78 & 1.06 \\
\hline 2D-FBP & Yes & 4.31 & 3.78 & 6.64 & 4.32 & 3.50 & 5.34 & 1.03 & 2.93 & 4.09 \\
\hline 2D-OSEM & No & 4.21 & 2.53 & 3.85 & 4.27 & 1.66 & 2.02 & 1.27 & 1.02 & 1.16 \\
\hline 2D-OSEM & Yes & 3.04 & 3.62 & 6.43 & 1.62 & 3.20 & 4.74 & 0.36 & 2.92 & 4.44 \\
\hline
\end{tabular}


TABLE 2

Percentage Error in Measured Ratio of Source Activities

\begin{tabular}{|c|c|c|c|c|c|c|c|}
\hline \multirow[b]{3}{*}{ Reconstruction } & \multirow[b]{3}{*}{ Smoothed } & \multicolumn{3}{|c|}{$220 \mathrm{~Bq}: 940 \mathrm{~Bq}$} & \multicolumn{3}{|c|}{$55 \mathrm{~Bq}: 940 \mathrm{~Bq}$} \\
\hline & & \multicolumn{3}{|c|}{ LLD (keV) } & \multicolumn{3}{|c|}{ LLD (keV) } \\
\hline & & 250 & 350 & 400 & 250 & 350 & 400 \\
\hline 2D-FBP & No & 29.2 & -5.2 & 1.5 & 108.6 & 29.1 & -0.5 \\
\hline 2D-FBP & Yes & 32.8 & 0.6 & 3.7 & 134.7 & 25.9 & 3.5 \\
\hline 2D-OSEM & No & 27.1 & -10.9 & -4.4 & 111.8 & 11.0 & -22.3 \\
\hline 2D-OSEM & Yes & 29.1 & -3.3 & -1.6 & 130.8 & 5.4 & -17.6 \\
\hline
\end{tabular}

\section{ACKNOWLEDGMENTS}

We gratefully acknowledge Pedro Rosa-Neto of the Montreal Neurological Institute and Shadreck Mzengeza of the Winnipeg Health Sciences Centre for their help with this work. This work was supported by grant OPG:0033672 from the Natural Science and Engineering Council of Canada and by the Winnipeg Health Sciences Centre.

\section{REFERENCES}

1. Dahlbom M, Cherry SR, Eriksson L, Hoffman EJ, Wienhard K. Optimization of PET instrumentation for brain activation studies. IEEE Trans Nucl Sci. 1993;40: 1048-1054.

2. Strother SC, Casey ME, Hoffman EJ. Measuring PET scanner sensitivity: relating count rates to image signal-to-noise ratios using noise equivalent counts. IEEE Trans Nucl Sci. 1990;37:783-788.

3. Turkington TG, Wilson JW, Colsher JG. Adjusting the low energy threshold for large bodies in PET. 2004 IEEE Nuclear Science Symposium Conference Record. 2004;5:2872-2876.

4. Watson CC, Casey ME, Bendriem B, et al. Optimizing injected dose in clinical PET by accurately modeling the counting-rate response functions specific to individual patient scans. J Nucl Med. 2005;46:1825-1834.

5. Adonai $\mathrm{N}$, Nguyen $\mathrm{KN}$, Walsh $\mathrm{J}$, et al. Ex vivo cell labeling with ${ }^{64} \mathrm{Cu}-$ pyruvaldehyde-bis(N4-methylthiosemicarbazone) for imaging cell trafficking in mice with positron-emission tomography. Proc Natl Acad Sci USA. 2002;99: 3030-3035.

6. Sundaresan G, Yazaki PJ, Shively JE, et al. ${ }^{124} \mathrm{I}$-Labeled engineered anti-CEA minibodies and diabodies allow high-contrast, antigen-specific smallanimal PET imaging of xenografts in athymic mice. J Nucl Med. 2003;44: 1962-1969.

7. Humm JL, Rosenfeld A, Del Guerra A. From PET detectors to PET scanners. Eur J Nucl Med Mol Imaging. 2003;30:1574-1597.

8. Eriksson L, Watson CC, Wienhard K, et al. The ECAT HRRT: an example of NEMA scatter estimation issues for LSO-based PET systems. IEEE Trans Nucl Sci. 2005;52:90-94.

9. Watson CC, Casey ME, Eriksson L, Mulnix T, Adams D, Bendriem B. NEMA NU 2 performance tests for scanners with intrinsic radioactivity. J Nucl Med. 2004;45:822-826.

10. Yamamoto S, Horii H, Hurutani M, Matsumoto K, Senda M. Investigation of single, random, and true counts from natural radioactivity in LSO-based clinical PET. Ann Nucl Med. 2005;19:109-114.

11. Browne E, Junde H. Nuclear data sheets for $\mathrm{A}=176$. Nucl Data Sheets. 1998;84:337-486.

12. Knoess C, Siegel S, Smith A, et al. Performance evaluation of the microPET R4 PET scanner for rodents. Eur J Nucl Med Mol Imaging. 2003;30:737747.

13. Laforest R, Longford D, Siegel S, Newport DF, Yap J. Performance evaluation of the microPET-Focus - F120. 2004 IEEE Nuclear Science Symposium Conference Record. 2004;5:2965-2969.

14. Daube-Witherspoon ME, Karp JS, Casey ME, et al. PET performance measurements using the NEMA NU 2-2001 standard. J Nucl Med. 2002;43:13981409 . 